\title{
Characterization of $V\left(\mathbb{Z} C_{n}^{+}\right)$of rank $\leq 4$
}

\author{
Bülent Köklüce and Nesibe Tüfekçi
}




\title{
CHARACTERIZATION OF $V\left(\mathbb{Z} \mathbf{C}_{n}^{+}\right)$OF RANK $\leq 4$
}

\author{
BÜLENT KÖKLÜCE AND NESIBE TÜFEKÇI
}

Received 24 September, 2013

\begin{abstract}
There are only a few cases for cyclic groups $\mathbf{C}_{n}$, where the unit group of their integral group rings are determined. In this article, we have completed the characterization of torsionfree part $V\left(\mathbb{Z} \mathbf{C}_{n}^{+}\right)$of the integral group ring of cyclic group $\mathbf{C}_{n}$, for $n=11,15,16,20,24$ and 30 where $\rho \leq 4$. We explicitly find all the generators.
\end{abstract}

2010 Mathematics Subject Classification: 16S34; 16 U60

Keywords: group ring, unit group, normalizer, generator

\section{INTRODUCTION}

Let $G$ be a finite group and $U(\mathbb{Z} G)$ be the unit group of integral group ring $\mathbb{Z} G$. We denote the group of normalized units of $\mathbb{Z} G$ by $\mathcal{U}=V(\mathbb{Z} G)$ and the subgroup of the central units of $\mathcal{U}$ by $\mathcal{Z}(\mathcal{U})$. By $\mathcal{N} U(G)$ we mean the normalizer of $G$ in $\mathcal{U}$. The structure of the unit group $\mathcal{U}(\mathbb{Z} G)$ has been of a fundamental interest after the G. Higman's thesis written in 1939. Problem 43 in [17] asks whether the normalizer property; $\mathcal{N} u(G)=G \mathcal{Z}(\mathcal{U})$, holds for any finite group $G$.

In [5] it is shown by Coleman in particular that, this property holds for any finite nilpotent group. Jackowski and Marciniak [8] extended this to finite groups of odd order and the groups having normal Sylow 2-subgroup. Later in [14] Li et al. have shown that if the intersection of non-normal subgroups of $G$ is non-trivial then, $G$ satisfies the normalizer property. Meanwhile, the normalizer property is verified by Li [13] for some metabelian groups and by Marciniak and Roggenkamp [15] for finite metabelian groups with an abelian Sylow 2-subgroup. We refer the reader to [2] for a general information about the theory of group rings and to $[6,7,9,11,16]$ for a survey on results on the unit group of integral group rings.

An explicit characterization of the normalizer of $D_{n}=\left\langle a, b: a^{n}=b^{2}=1, b^{-1} a b=\right.$ $\left.a^{-1}\right\rangle$, dihedral group of order $2 n$, in the normalized units of $\mathbb{Z} D_{n}$ has recently been given by Bilgin [4]. In the present paper, we give the characterization of torsionfree part of the integral group ring of some cyclic groups. Before we give the main theorems we obtained, we introduce some notations and give some basic facts. 
Let $\mathbf{C}_{n}=<a: a^{n}=1>$ be a cyclic group of order $n, \mathbb{Z} \mathbf{C}_{n}$ be its integral group ring and $V\left(\mathbb{Z} \mathbf{C}_{n}\right)$ be its normalized units. Now, let us consider the following subring of symmetric elements of $\mathbb{Z} \mathbf{C}_{n}$

$$
\mathbb{Z C}_{n}^{+}=\left\{\gamma=\sum_{i=0}^{n-1} \gamma_{i} a^{i}: \gamma_{i}=\gamma_{n-i}\right\} .
$$

If we denote the unit group of $\mathbb{Z} \mathbf{C}_{n}^{+}$by $U\left(\mathbb{Z} \mathbf{C}_{n}^{+}\right)$and its normalized units by $V($ $\mathbb{Z} \mathbf{C}_{n}^{+}$) then we can state Higman's following result :

Theorem 1. $\mathcal{N} u\left(D_{n}\right)=D_{n} \times V\left(\mathbb{Z} \mathbf{C}_{n}^{+}\right)$.

When we modify Higman's result [17] for a cyclic group of order $n$ instead of a finite abelian group, we obtain the following corollary:

Corollary 1. $V\left(\mathbb{Z} \mathbf{C}_{n}^{+}\right)=\mathcal{Z}\left(D_{n}\right) \times F$, where $F$ is a free abelian group of rank

$$
\rho=\frac{1}{2} \varphi(n)-1 \text {. }
$$

Corollary 1 reduces the characterization of $V\left(\mathbb{Z} \mathbf{C}_{n}\right)$ to the construction of generator(s) of $F$. So, $V\left(\mathbb{Z} \mathbf{C}_{n}\right)$ is trivial when $n=1,2,3,4$ or 6 . Torsion free part of $V\left(\mathbb{Z} \mathbf{C}_{n}\right)$ has a unique generator if $n=5,8,10$ or 12 , and it has been characterized for $n=5,8$ and 12 in $[3,10]$ as follows

$$
\begin{gathered}
V\left(\mathbb{Z} \mathbf{C}_{5}\right)=\mathbf{C}_{5} \times\left\langle-1+a+a^{4}\right\rangle, \\
V\left(\mathbb{Z} \mathbf{C}_{8}\right)=\mathbf{C}_{8} \times\left\langle-1-\left(a+a^{-1}\right)+\left(a^{3}+a^{-3}\right)+2 a^{4}\right\rangle,
\end{gathered}
$$

$$
\begin{aligned}
& V\left(\mathbb{Z} \mathbf{C}_{12}\right)=\mathbf{C}_{12} \\
& \quad \times\left\langle 3+2\left(a+a^{-1}\right)+\left(a^{2}+a^{-2}\right)-\left(a^{4}+a^{-4}\right)-2\left(a^{5}+a^{-5}\right)-2 a^{6}\right\rangle .
\end{aligned}
$$

$\rho=2$ when $n=7,9,14$ or 18 . For $n=7, F$ was characterized by Karpilovsky [10] and for the cases $n=7$ and 9, it was characterized by Aleev [1]. A different characterization for $n=7$ and 9 was also given by Kokluce and Kelebek [12] as

$$
\begin{aligned}
V\left(\mathbb{Z} \mathbf{C}_{7}\right)= & \mathbf{C}_{7} \times\left\langle-1+\left(a+a^{-1}\right)\right\rangle \times\left\langle-1+2\left(a+a^{-1}\right)-\left(a^{2}+a^{-2}\right)\right\rangle, \\
V\left(\mathbb{Z} \mathbf{C}_{9}\right)= & \mathbf{C}_{9} \times\left\langle-1+\left(a+a^{-1}\right)-\left(a^{4}+a^{-4}\right)\right\rangle \\
& \times\left\langle-1+\left(a+a^{-1}\right)+\left(a^{2}+a^{-2}\right)+\left(a^{3}+a^{-3}\right)-2\left(a^{4}+a^{-4}\right)\right\rangle .
\end{aligned}
$$

There is a strong relationship between $F$ and $V\left(\mathbb{Z} \mathbf{C}_{n}^{+}\right)$. It is clear that $V\left(\mathbb{Z} \mathbf{C}_{n}^{+}\right)$ and $F$ have the same rank. In some cases they are the same. Thus the characterization of $V\left(\mathbb{Z} \mathbf{C}_{n}^{+}\right)$encourages one to make the characterization of $V\left(\mathbb{Z} \mathbf{C}_{n}\right)$. 
In this study, we have completed the characterization of normalizers of dihedral groups where the rank $\rho \leq 4$. The following table lists the orders of dihedral groups corresponding to rank $\rho \leq 4$.

TABLE 1.

\begin{tabular}{|l|l|}
\hline $\operatorname{rank}(\rho)$ & $\operatorname{order}(n)$ \\
\hline 0 & $1,2,3,4,6$ \\
\hline 1 & $5,8,10,12$ \\
\hline 2 & $7,9,14,18$ \\
\hline 3 & $15,16,20,24,30$ \\
\hline 4 & 11,22 \\
\hline
\end{tabular}

Let $\omega=e^{\frac{2 \pi i}{n}}$ and $\alpha=\omega+\omega^{-1}$. The minimal polynomial $\min Q_{Q}(\alpha, x)$ of $\alpha$ over $Q$ can be obtained by a simple calculation. The image of $\mathbb{Z} \mathbf{C}_{n}^{+}$under the ring homomorphism

$$
\psi: \begin{aligned}
\mathbb{Z} \mathbf{C}_{n} & \rightarrow \mathbb{Z}[] \\
\sum \gamma_{i} a^{i} & \mapsto \sum \gamma_{i} \omega^{i}
\end{aligned}
$$

gives the ring of integers $\mathbb{Z}[]$. In determination of the normalized units $V\left(\mathbb{Z} \mathbf{C}_{n}^{+}\right)$of $\mathbb{Z} \mathbf{C}_{n}^{+}$we firstly need to find the fundamental units of $\mathbb{Z}[]$ whose minimal polynomial is $\min _{Q}(\alpha, x)$. The following Table 2 list the all fundamental units of $\mathbb{Z}[]$ for necessary cases.

TABLE 2.

\begin{tabular}{|l|l|l|l|}
\hline$\rho$ & $n$ & $\min _{Q}(\alpha, x)$ & Fundamental Units \\
\hline \multirow{3}{*}{3} & \multirow{2}{*}{15} & $x^{4}-x^{3}-4 x^{2}+4 x+1$ & $\varepsilon_{1}=\alpha-1$ \\
& & & $\varepsilon_{2}=\alpha^{2}-3$ \\
$\varepsilon_{3}=\alpha^{3}-3 \alpha$ \\
\hline \multirow{3}{*}{3} & \multirow{2}{*}{16} & $x^{4}-4 x^{2}+2$ & $\varepsilon_{1}=\alpha-1$ \\
& & & $\varepsilon_{2}=\alpha^{2}-1$ \\
& & $\varepsilon_{3}=\alpha^{2}+\alpha-1$ \\
\hline \multirow{3}{*}{3} & 20 & \multirow{2}{*}{$x^{4}-5 x^{2}+5$} & $\varepsilon_{1}=\alpha^{2}-2$ \\
& & & $\varepsilon_{2}=\alpha^{2}+\alpha-2$ \\
& & & $\varepsilon_{3}=\alpha^{3}-\alpha^{2}-3 \alpha+3$ \\
& & & $\varepsilon_{1}=\alpha$ \\
4 & 11 & $x^{5}+x^{4}-4 x^{3}-3 x^{2}+3 x+1$ & $\varepsilon_{2}=\alpha+1$ \\
& & & $\varepsilon_{3}=\alpha^{2}-2$ \\
& & & $\varepsilon_{4}=\alpha^{4}+\alpha^{3}-3 \alpha^{2}-3 \alpha$ \\
\hline
\end{tabular}




\section{Motivation for Contruction of $V\left(\mathbb{Z} \mathbf{C}_{n}^{+}\right)$.}

Lemma 1. Any $\gamma \in V\left(\mathbb{Z} \mathbf{C}_{n}^{+}\right)$can be written as

$$
\gamma=\gamma_{0}+\sum_{i=1}^{k} \gamma_{i} C_{i}
$$

where $C_{i}=a^{i}+a^{-i}$.

Proof. Let us denote $C_{i}=a^{i}+a^{-i}$ and $\gamma \in V\left(\mathbb{Z} \mathbf{C}_{n}^{+}\right)$. If $n=2 k+1$ then $\gamma=$ $\gamma_{0}+\sum_{i=1}^{k} \gamma_{i} C_{i}$. If $n=2 k$ then $\gamma=\gamma_{0}+\gamma_{k}^{\prime} a^{k}+\sum_{i=1}^{k-1} \gamma_{i} C_{i}$. By considering their augmentations, we have

$$
\varepsilon(\gamma)=\left\{\begin{aligned}
\gamma_{0}+2 \sum_{i=1}^{k} \gamma_{i} & , n=2 k+1 \\
\gamma_{0}+\gamma_{k}^{\prime}+2 \sum_{i=1}^{k-1} \gamma_{i}, & n=2 k .
\end{aligned}\right.
$$

by modulo 2 ,we obtain

$$
\varepsilon(\gamma) \equiv 1(\bmod 2) \Rightarrow\left\{\begin{array}{c}
\gamma_{0} \equiv 1(\bmod 2), n=2 k+1 \\
\gamma_{0}+\gamma_{k}^{\prime} \equiv 1(\bmod 2), n=2 k
\end{array}\right.
$$

By choosing $\gamma_{0}$ as an odd integer in both cases we obtain $\gamma_{k}^{\prime}=2 \gamma_{k}$, for some $\gamma_{k} \in \mathbb{Z}$ we have

$$
\gamma_{k}^{\prime} a^{k}=2 \gamma_{k} a^{k}=\gamma_{k}\left(a^{k}+a^{-k}\right)=\gamma_{k} C_{k} .
$$

So, $\gamma \in V\left(\mathbb{Z} \mathbf{C}_{n}^{+}\right)$can be written as

$$
\gamma=\gamma_{0}+\sum_{i=1}^{k} \gamma_{i} C_{i}
$$

in both cases.

Proposition 1. Let $H$ be a subgroup of a finite abelian group $G$. We can define a group epimorphism:

$$
\begin{aligned}
\varphi: \quad & G \rightarrow G / H \\
& g \mapsto g H .
\end{aligned}
$$

If we extend $\varphi$ linearly over $\mathbb{Z}$, then we can get the natural ring homomorphism

$$
\begin{aligned}
\bar{\varphi}: \quad \mathbb{Z} G & \rightarrow \mathbb{Z}(G / H) \\
\sum \gamma_{g} g & \mapsto \sum \gamma_{g}(g H) .
\end{aligned}
$$

If $G / H \cong \mathbf{C}_{2}, \mathbf{C}_{3}, \mathbf{C}_{4}$ or $\mathbf{C}_{6}$, then for any torsion-free unit $\gamma \in V(\mathbb{Z} G), \bar{\varphi}(\gamma)=H$.

Remark 1. If $\gamma \in V\left(\mathbb{Z} \mathbf{C}_{n}^{+}\right)$and $n=2 k$ then $a^{k} \gamma \in V\left(\mathbb{Z} \mathbf{C}_{n}^{+}\right)$. The coefficient of identity of either $\gamma$ or $a^{k} \gamma$ is odd. $\gamma \in V\left(\mathbb{Z} \mathbf{C}_{n}^{+}\right)$can be chosen as a generator if the coefficient of its identity is odd. 
Proposition 2. Let $n$ be an odd integer then $\mathbf{C}_{2 n}=<a: a^{2 n}=1>$ and $H=<$ $a^{2}>$. Then $V\left(\mathbb{Z} \mathbf{C}_{n}^{+}\right)$and $V\left(\mathbb{Z} H^{+}\right)$have the same rank.

Proof. If we extend group epimorphism linearly over $\mathbb{Z}$

$$
\begin{aligned}
f: \quad & \mathbf{C}_{2 n} \rightarrow H \\
& a^{i} \mapsto a^{2 i} .
\end{aligned}
$$

then, we obtain the following ring epimorphism:

$$
\begin{aligned}
\bar{f}: & \mathbb{Z} \mathbf{C}_{2 n} \rightarrow \mathbb{Z} H \\
& \sum_{i=0}^{2 n-1} \gamma_{i} a^{i} \mapsto \sum_{i=0}^{2 n-1} \gamma_{i} a^{2 i} .
\end{aligned}
$$

If we restrict $\bar{f}$ to multiplicative torsion-free group we have

$$
\widetilde{f}: \quad V\left(\mathbb{Z} \mathbf{C}_{n}^{+}\right) \rightarrow V\left(\mathbb{Z} H^{+}\right) .
$$

Since

$$
\begin{aligned}
\rho_{2 n} & =\frac{1}{2} \varphi(2 n)-1 \\
& =\frac{1}{2} \varphi(2) \varphi(n)-1 \\
& =\frac{1}{2} \varphi(n)-1 \\
& =\rho_{n},
\end{aligned}
$$

$V\left(\mathbb{Z} \mathbf{C}_{n}^{+}\right)$and $V\left(\mathbb{Z} H^{+}\right)$have the same rank.

Remark 2. Let be $n$ be an odd integer. If $\gamma=\gamma_{0}+\sum_{i=0}^{k} \gamma_{i} C_{i}$ is a generator of $V\left(\mathbb{Z} H^{+}\right) \subset \mathbb{Z} \mathbf{C}_{2 n}$, then by Proposition $2 \gamma=\gamma_{0}+\sum_{i=0}^{k} \gamma_{i} a^{2 i}$ is a generator of $V\left(\mathbb{Z} \mathbf{C}_{n}^{+}\right)$.

By using Remark 2 and considering (1.1), (1.4) and (1.5), the unit groups $V\left(\mathbb{Z} \mathbf{C}_{10}\right)$, $V\left(\mathbb{Z} \mathbf{C}_{14}\right)$ and $V\left(\mathbb{Z} \mathbf{C}_{18}\right)$ can as follows be given as follows:

$$
\begin{gathered}
V\left(\mathbb{Z} \mathbf{C}_{10}\right)=\mathbf{C}_{10} \times\left\langle-1+a^{2}+a^{8}\right\rangle, \\
V\left(\mathbb{Z} \mathbf{C}_{14}\right)=\mathbf{C}_{14} \times\left\langle-1+\left(a^{2}+a^{-2}\right)\right\rangle \times\left\langle-1+2\left(a^{2}+a^{-2}\right)-\left(a^{4}+a^{-4}\right)\right\rangle, \\
V\left(\mathbb{Z} \mathbf{C}_{18}\right)=\mathbf{C}_{18} \times\left\langle-1+\left(a^{2}+a^{-2}\right)-\left(a^{8}+a^{-8}\right)\right\rangle \\
\times\left\langle-1+\left(a^{2}+a^{-2}\right)+\left(a^{4}+a^{-4}\right)+\left(a^{6}+a^{-6}\right)-2\left(a^{8}+a^{-8}\right)\right\rangle .
\end{gathered}
$$




\section{Characterization of $V\left(\mathbb{Z} \mathbf{C}_{n}^{+}\right)$.}

\section{Characterization of $V\left(\mathbb{Z} \mathbf{C}_{15}^{+}\right)$and $V\left(\mathbb{Z} \mathbf{C}_{30}^{+}\right)$}

\section{Theorem 2.}

$V\left(\mathbb{Z}_{15}^{+}\right)=<-1+C_{1}-C_{2}+C_{3}-C_{4}+C_{5},-1+C_{2}-C_{4}+C_{5}+C_{6}-C_{7},-1+C_{3}>$.

Proof. Let $\gamma \in V\left(\mathbb{Z} \mathbf{C}_{15}^{+}\right)$be a generator of torsion-free unit. Then, by Lemma 1 we have

$$
\gamma=\gamma_{0}+\sum_{i=1}^{7} \gamma_{i} C_{i} .
$$

Let us consider the subgroups $H_{1}=\left\langle a^{3}\right\rangle$ and $H_{2}=\left\langle a^{5}\right\rangle$ of prime order. Since $\mathbf{C}_{15} / H_{1} \cong \mathbf{C}_{3}$ and $\mathbf{C}_{15} / H_{2} \cong \mathbf{C}_{5}$ by Propositon 1 , we have $\bar{\varphi}_{j}(\gamma)=H_{j}(j=1,2)$. For $\bar{\varphi}_{1}(\gamma)=H_{1}$, we obtain

$$
\begin{aligned}
\gamma_{0}+2 \gamma_{3}+2 \gamma_{6} & =1, \\
\gamma_{1}+\gamma_{2}+\gamma_{4}+\gamma_{5}+\gamma_{7} & =0 .
\end{aligned}
$$

and for $\bar{\varphi}_{2}(\gamma)=H_{2}$, we obtain

$$
\begin{aligned}
\gamma_{0}+2 \gamma_{5} & =1, \\
\gamma_{1}+\gamma_{4}+\gamma_{6} & =0, \\
\gamma_{2}+\gamma_{3}+\gamma_{7} & =0 .
\end{aligned}
$$

Substituting $\gamma_{1}=p, \gamma_{2}=q, \gamma_{4}=r, \gamma_{7}=s$ in (3.1) and (3.2), we obtain

$$
\begin{aligned}
& \gamma_{0}=1+2(p+q+r+s), \\
& \gamma_{3}=-q-s, \\
& \gamma_{5}=-(p+q+r+s), \\
& \gamma_{6}=-p-r .
\end{aligned}
$$

Denoting $\omega=e^{\frac{2 \pi i}{15}}$ and $\alpha=\omega+\omega^{-1}$ we get the minimal polynomial of $\alpha$ over $\mathbb{Q}$ as follows:

$$
\min _{\mathbb{Q}}(\alpha, x)=x^{4}-x^{3}-4 x^{2}+4 x+1 .
$$

Now, let us consider the following ring homomorphism:

$$
\psi: \begin{aligned}
\mathbb{Z} \mathbf{C}_{15} & \rightarrow \mathbb{Z}[] \\
\sum \gamma_{i} a^{i} & \mapsto \sum \gamma_{i} \omega^{i} .
\end{aligned}
$$

By (3.3) and (3.4), the image of the unit is

$$
\begin{aligned}
\psi(\gamma) & =\psi\left(\gamma_{0}+\sum_{i=1}^{7} \gamma_{i} C_{i}\right) \\
& =\gamma_{0}+\gamma_{1}(\alpha)+\gamma_{2}\left(\alpha^{2}-2\right)+\gamma_{3}\left(\alpha^{3}-3 \alpha\right)+\gamma_{4}\left(\alpha^{3}-4 \alpha+1\right)
\end{aligned}
$$




$$
\begin{aligned}
& +\gamma_{5}(-1)+\gamma_{6}\left(-\alpha^{3}+3 \alpha-1\right)+\gamma_{7}\left(-\alpha^{3}-\alpha^{2}+3 \alpha+2\right) \\
& =(1+4 p+q+5 r+5 s)+(-2 p+3 q-7 r+6 s) \alpha+(q-s) \alpha^{2} \\
& +(p-q+2 r-2 s) \alpha^{3}
\end{aligned}
$$

Since $\psi(\gamma) \in \mathcal{U}(\mathbb{Z}[\alpha])=\left\{\sum_{i=0}^{3} a_{i} \alpha^{i}: \alpha^{4}-\alpha^{3}-4 \alpha^{2}+4 \alpha+1=0\right\}$ by Table 2 ,

$$
u(\mathbb{Z}[\alpha])=\left\langle\alpha-1, \alpha^{2}-3, \alpha^{3}-3 \alpha\right\rangle .
$$

As a consequence of the calculations we see that $\gamma \in U\left(\mathbb{Z}\left[a+a^{-1}\right]\right)$ can be obtained in the following single product of the fundamental units or their inverses;

$$
\varepsilon_{1}^{2}, \varepsilon_{2}^{2}, \varepsilon_{1}^{-2}, \varepsilon_{2}^{-2} \text {. }
$$

One can easily see that

$$
\begin{aligned}
& \psi(\gamma)=\varepsilon_{1}^{2} \Rightarrow \gamma=-1+C_{1}-C_{2}+C_{3}-C_{4}+C_{5}, \\
& \psi(\gamma)=\varepsilon_{2}^{2} \Rightarrow \gamma=-1+C_{2}-C_{4}+C_{5}+C_{6}-C_{7} .
\end{aligned}
$$

Since $H_{1}=\left\langle a^{3}\right\rangle$ is a cyclic group of order 5 , by (1.1), its unit group of integral group ring $V\left(\mathbb{Z} H_{1}\right)=H_{1} \times\left\langle-1+\left(a^{3}+a^{-3}\right)\right\rangle$, so the third unit is $-1+C_{3}$.

Corollary 2.

$$
=<-1+C_{2}-C_{4}+C_{5}+C_{6}-C_{7},-1-C_{1}+C_{3}+C_{4}+C_{5}-C_{7},-1+C_{6}>.
$$

Proof. By considering Theorem 2 with Remark 2 we get the required result.

\section{Characterization of $V\left(\mathbb{Z} \mathbf{C}_{16}^{+}\right)$}

\section{Theorem 3.}

$V\left(\mathbb{Z} \mathbf{C}_{16}^{+}\right)=<1-C_{2}-C_{3}+C_{5}+C_{6}, 1-C_{1}+C_{2}-C_{6}+C_{7},-1-C_{2}+C_{6}+C_{8}>$

Proof. Let $\gamma \in V\left(\mathbb{Z} \mathbf{C}_{16}^{+}\right)$be a generator of torsion-free unit. Then, by Lemma 1 we have

$$
\gamma=\gamma_{0}+\sum_{i=1}^{8} \gamma_{i} C_{i}
$$

Let us consider the prime subgroup $H=\left\langle a^{8}\right\rangle$. Since $\mathbf{C}_{16} / H \cong \mathbf{C}_{8}$ by Propositon $1 \mathcal{U}\left(\mathbb{Z}\left(\mathbf{C}_{16} / H\right)\right)$ is trivial. For any $\gamma \in V\left(\mathbb{Z} \mathbf{C}_{16}^{+}\right)$we get

$$
\begin{aligned}
\bar{\varphi}(\gamma) & =H \Rightarrow \gamma_{0}+\gamma_{8}=1, \\
\gamma_{i}+\gamma_{8-i} & =0 \text { for } i=1,2,3,4 .
\end{aligned}
$$

Substituting $\gamma_{0}=p, \gamma_{1}=q, \gamma_{2}=r, \gamma$ in (3.5), we have

$$
\gamma_{4}=0, \gamma_{5}=-s, \gamma_{6}=-r, \gamma_{7}=-q, \gamma_{8}=1-p \text {. }
$$


Denote $\omega=e^{\frac{2 \pi i}{16}}$ and $\alpha=\omega+\omega^{-1}$ then the minimal polynomial of $\alpha$ over $\mathbb{Q}$ can be obtained as follows

$$
\min _{\mathbb{Q}}(\alpha, x)=x^{4}-4 x^{2}+2 .
$$

By considering the following ring homomorphism

$$
\begin{aligned}
\psi: \quad \mathbb{Z}_{16} & \rightarrow \mathbb{Z}[] \\
\sum \gamma_{i} a^{i} & \mapsto \sum \gamma_{i} \omega^{i},
\end{aligned}
$$

and (3.6), the image of the unit can be given as

$$
\begin{aligned}
\psi(\gamma) & =\psi\left(\gamma_{0}+\sum_{i=1}^{8} \gamma_{i} C_{i}\right) \\
& =\gamma_{0}+\gamma_{1}(\alpha)+\gamma_{2}\left(\alpha^{2}-2\right)+\gamma_{3}\left(\alpha^{3}-3 \alpha\right)+\gamma_{4}(0) \\
& +\gamma_{5}\left(-\alpha^{3}+3 \alpha\right)+\gamma_{6}\left(-\alpha^{2}+2\right)+\gamma_{7}(-\alpha)-\gamma_{8} \\
& =(-1+2 p-4 r)+(2 q-6 s) \alpha+(2 r) \alpha^{2}+(2 s) \alpha^{3} .
\end{aligned}
$$

Since $\psi(\gamma) \in \mathcal{U}(\mathbb{Z}[\alpha])=\left\{\sum_{i=0}^{4} a_{i} \alpha^{i}: \alpha^{4}-4 \alpha^{2}+2=0\right\}$, by Table 2 we have

$$
u(\mathbb{Z}[\alpha])=\left\langle\alpha-1, \alpha^{2}-1, \alpha^{2}+\alpha-1\right\rangle .
$$

The calculations shows that $\gamma \in U\left(\mathbb{Z}\left[a+a^{-1}\right]\right)$ can be obtained in the following double products of the fundamental units or their inverses;

$$
\varepsilon_{1}^{2} \varepsilon_{2}^{-1}, \varepsilon_{1}^{2} \varepsilon_{2}, \varepsilon_{1}^{-2} \varepsilon_{2}, \varepsilon_{1}^{-2} \varepsilon_{2}^{-1}, \varepsilon_{3}^{2} \varepsilon_{2}, \varepsilon_{3}^{-2} \varepsilon_{2}, \varepsilon_{3}^{-2} \varepsilon_{2}^{-1}, \varepsilon_{3}^{2} \varepsilon_{2}^{-1} .
$$

One can easily see that these units can be generated three units. The first one can be chosen as

$$
\psi(\gamma)=\varepsilon_{2} \varepsilon_{1}^{-2} \Rightarrow \gamma=C_{2}-C_{3}+C_{5}-C_{6}+a^{8} .
$$

By considering Remark 1 we may write the first unit as follows

$$
a^{8} \gamma=1-C_{2}-C_{3}+C_{5}+C_{6}
$$

The second generator is

$$
\psi(\gamma)=\varepsilon_{2} \varepsilon_{3}^{-2} \Rightarrow \gamma=1-C_{1}+C_{2}-C_{6}+C_{7} .
$$

Since $K=\left\langle a^{2}\right\rangle$ is a cyclic group of order 8, by (1.2), its unit group of integral group ring $V(\mathbb{Z} K)=K \times\left\langle-1-\left(a^{2}+a^{-2}\right)+\left(a^{6}+a^{-6}\right)+2 a^{8}\right\rangle$, so the third generator is $-1-C_{2}+C_{6}+2 a^{8}$. By Lemma 1 , the third generator can be written as $-1-C_{2}+$ $C_{6}+C_{8}$ which finishes the proof. 


\section{Characterization of $V\left(\mathbb{Z} \mathbf{C}_{20}^{+}\right)$}

\section{Theorem 4.}

$$
\begin{gathered}
V\left(\mathbb{Z} \mathbf{C}_{20}^{+}\right)=<1+C_{1}+C_{2}+C_{3}-C_{7}-C_{8}-C_{9}-C_{10}, \\
1-C_{1}+C_{3}-C_{4}+C_{6}-C_{7}+C_{9}-C_{10},-1+C_{4}>
\end{gathered}
$$

Proof. Let $\gamma \in V\left(\mathbb{Z} \mathbf{C}_{20}^{+}\right)$be a generator of torsion-free unit. By Lemma 1 we have

$$
\gamma=\gamma_{0}+\sum_{i=1}^{10} \gamma_{i} C_{i} .
$$

Let us consider subgroups $H_{1}=\left\langle a^{10}\right\rangle$ and $H_{2}=\left\langle a^{4}\right\rangle$ of prime indices. Since $\mathbf{C}_{20} / H_{1} \cong \mathbf{C}_{10}$ and $\mathbf{C}_{20} / H_{2} \cong \mathbf{C}_{4}$ by Propositon 1 we have $\bar{\varphi}_{j}(\gamma)=H_{j}(j=$ 1,2).For $\bar{\varphi}_{1}(\gamma)=H_{1}$, we obtain

$$
\begin{aligned}
\gamma_{0}+\gamma_{10} & =1, \quad \\
\gamma_{i}+\gamma_{10-i} & =0, \quad(i=1,2,3,4,5) .
\end{aligned}
$$

and for $\bar{\varphi}_{2}(\gamma)=H_{2}$, we get

$$
\begin{aligned}
\gamma_{0}+2 \gamma_{4}+2 \gamma_{8} & =1, \\
\gamma_{1}+\gamma_{3}+\gamma_{5}+\gamma_{7}+\gamma_{9} & =0, \\
2 \gamma_{2}+2 \gamma_{6}+\gamma_{10} & =0 .
\end{aligned}
$$

If we substitude $\gamma_{1}=p, \gamma_{2}=q, \gamma_{3}=r, \gamma_{4}=s$ in (3.7) and (3.8), we can write

$$
\begin{aligned}
& \gamma_{0}=1+2 q-2 s, \gamma_{5}=0, \\
& \gamma_{6}=-s, \gamma_{7}=-r, \gamma_{8}=-q, \\
& \gamma_{9}=-p, \gamma_{10}=-2 q+2 s .
\end{aligned}
$$

By taking $\omega=e^{\frac{2 \pi i}{20}}$ and $\alpha=\omega+\omega^{-1}$ we can write the minimal polynomial of $\alpha$ over $\mathbb{Q}$ as follows;

$$
\min _{\mathbb{Q}}(\alpha, x)=x^{4}-5 x^{2}+5 .
$$

Now consider the following ring homomorphism,

$$
\psi: \begin{aligned}
\mathbb{Z} \mathbf{C}_{20} & \rightarrow \mathbb{Z}[] \\
\sum \gamma_{i} a^{i} & \mapsto \sum \gamma_{i} \omega^{i}
\end{aligned}
$$

with equations (3.9) and (3.10). This gives the image of the unit as

$$
\begin{aligned}
\psi(\gamma) & =\psi\left(\gamma_{0}+\sum_{i=1}^{10} \gamma_{i} C_{i}\right),\left(C_{10}=2 a^{3}\right) \\
& =\gamma_{0}+\gamma_{1}(\alpha)+\gamma_{2}\left(\alpha^{2}-2\right)+\gamma_{3}\left(\alpha^{3}-3 \alpha\right)+\gamma_{4}\left(\alpha^{4}-4 \alpha^{2}+2\right)+\gamma_{5}(0), \\
& +\gamma_{6}\left(-\alpha^{4}+4 \alpha^{2}-2\right)+\gamma_{7}\left(-\alpha^{3}+3 \alpha\right)+\gamma_{8}\left(-\alpha^{2}+2\right)+\gamma_{9}(-\alpha)-\gamma_{10}
\end{aligned}
$$




$$
=(1-10 s)+(2 p-6 r) \alpha+(2 q+2 s) \alpha^{2}+(2 r) \alpha^{3} .
$$

Since $\psi(\gamma) \in U(\mathbb{Z}[\alpha])=\left\{\sum_{i=0}^{4} a_{i} \alpha^{i}: \alpha^{4}-5 \alpha^{2}+5=0\right\}$. By using Table 2

$$
U(\mathbb{Z}[\alpha])=\left\langle\alpha^{2}-2, \alpha^{2}+\alpha-2, \alpha^{3}-\alpha^{2}-3 \alpha+3\right\rangle .
$$

$\gamma \in U\left(\mathbb{Z}\left[a+a^{-1}\right]\right)$ can be obtained in the following single product of the fundamental units or their inverses;

$$
\varepsilon_{2}^{2}, \varepsilon_{3}^{2}, \varepsilon_{2}^{-2}, \varepsilon_{3}^{-2} .
$$

The two generators are obtained as follows:

$$
\begin{aligned}
& \psi(\gamma)=\varepsilon_{2}^{2} \Rightarrow \gamma=-1-C_{1}-C_{2}-C_{3}+C_{7}+C_{8}+C_{9}+2 C_{10} \\
& \psi(\gamma)=\varepsilon_{3}^{2} \Rightarrow \gamma=-1+C_{1}-C_{3}+C_{4}-C_{6}+C_{7}-C_{9}+2 C_{10} .
\end{aligned}
$$

Their augmentations are negative, to make normalized units we must multiply by -1 and also by regarding Lemma 1 we get

$$
\begin{aligned}
& \psi(\gamma)=\varepsilon_{2}^{2} \Rightarrow \gamma=1+C_{1}+C_{2}+C_{3}-C_{7}-C_{8}-C_{9}-C_{10} \\
& \psi(\gamma)=\varepsilon_{3}^{2} \Rightarrow \gamma=1-C_{1}+C_{3}-C_{4}+C_{6}-C_{7}+C_{9}-C_{10} .
\end{aligned}
$$

Since $H_{2}=\left\langle a^{4}\right\rangle$ is a cyclic group of order 5 , by (1.1), its unit group of integral group ring $V\left(\mathbb{Z} H_{2}\right)=H_{2} \times\left\langle-1+\left(a^{4}+a^{-4}\right)\right\rangle$, so the third generator is clearly $-1+$ $C_{4}$.

\section{Characterization of $V\left(\mathbb{Z} \mathbf{C}_{24}^{+}\right)$}

\section{Theorem 5.}

$V\left(\mathbb{Z} \mathbf{C}_{24}^{+}\right)=<-5-2 C_{1}-4 C_{3}-3 C_{4}+2 C_{5}-2 C_{7}+3 C_{8}+4 C_{9}+2 C_{10}+3 C_{12}$, $3+2 C_{2}+C_{4}-C_{8}-2 C_{10}-C_{12},-1+C_{1}-C_{4}+C_{5}-C_{7}+C_{8}-C_{11}+C_{12}>$

Proof. Let $\gamma \in V\left(\mathbb{Z} \mathbf{C}_{24}^{+}\right)$be a generator of torsion-free unit. Then, by Lemma 1 we have

$$
\gamma=\gamma_{0}+\sum_{i=1}^{12} \gamma_{i} C_{i}
$$

Consider the subgroups $H_{1}=\left\langle a^{12}\right\rangle$ and $H_{2}=\left\langle a^{8}\right\rangle$ of prime orders. Since $\mathbf{C}_{24} / H_{1} \cong \mathbf{C}_{12}$ and $\mathbf{C}_{24} / H_{2} \cong \mathbf{C}_{8}$, by Propositon 1 we have, $\bar{\varphi}_{j}(\gamma)=H_{j}(j=$ $1,2)$.

For $\bar{\varphi}_{1}(\gamma)=H_{1}$, we obtain

$$
\begin{aligned}
\gamma_{0}+\gamma_{12} & =1, \quad(i=1,2,3,4,5,6) . \\
\gamma_{i}+\gamma_{12-i} & =0, \quad \text {. }
\end{aligned}
$$


and for $\bar{\varphi}_{2}(\gamma)=H_{2}$, we get

$$
\begin{aligned}
\gamma_{0}+2 \gamma_{8} & =1, \\
\gamma_{1}+\gamma_{7}+\gamma_{9} & =0, \\
\gamma_{2}+\gamma_{6}+\gamma_{10} & =0, \\
\gamma_{3}+\gamma_{5}+\gamma_{11} & =0, \\
2 \gamma_{4}+\gamma_{12} & =0 .
\end{aligned}
$$

Substituting $\gamma_{2}=p, \gamma_{3}=q, \gamma_{4}=r, \gamma_{5}=s$ in (3.11) and (3.12), we have

$$
\begin{array}{rlrl}
\gamma_{0} & =1+2 r, & \gamma_{1}=q+s, & \gamma_{6}=0 \\
\gamma_{7}=-s, & \gamma_{8}=-r, & \gamma_{9}=-q, \\
\gamma_{10}=-p, & \gamma_{11}=-q-s, & \gamma_{12}=-2 r
\end{array}
$$

On the other hand, if we denote $\omega=e^{\frac{2 \pi i}{24}}$ and $\alpha=\omega+\omega^{-1}=2 \cos \left(\frac{\pi}{12}\right)$ then we get the minimal polynomial of $\alpha$ over $\mathbb{Q}$ as

$$
\min _{\mathbb{Q}}(\alpha, x)=x^{4}-4 x^{2}+1 .
$$

Considering the ring homomorphism

$$
\psi: \begin{aligned}
\mathbb{Z} \mathbf{C}_{24} & \rightarrow \mathbb{Z}[] \\
\sum \gamma_{i} a^{i} & \mapsto \sum \gamma_{i} \omega^{i}
\end{aligned}
$$

with (3.13) and (3.14), the image of the unit can be obtained as

$$
\begin{aligned}
\psi(\gamma) & =\psi\left(\gamma_{0}+\sum_{i=1}^{11} \gamma_{i} C_{i}\right) \\
& =\left(\gamma_{0}-\gamma_{12}\right)+\left(\gamma_{1}-\gamma_{11}\right)\left(\frac{\sqrt{6}+\sqrt{2}}{2}\right)+\left(\gamma_{2}-\gamma_{10}\right)(\sqrt{3}) \\
& +\left(\gamma_{3}-\gamma_{9}\right)(\sqrt{2})+\left(\gamma_{4}-\gamma_{8}\right)(1)+\left(\gamma_{5}-\gamma_{7}\right)\left(\frac{\sqrt{6}-\sqrt{2}}{2}\right) \\
& =(1+6 r)+3 q \sqrt{2}+2 p \sqrt{3}+(q+2 s) \sqrt{6} .
\end{aligned}
$$

Since $\psi(\gamma) \in \mathcal{U}(\mathbb{Z}[\sqrt{2}]) \times U(\mathbb{Z}[\sqrt{3}]) \times \mathcal{U}(\mathbb{Z}[\sqrt{6}])$,

$$
\text { i) } \begin{aligned}
\psi(\gamma) \in \mathcal{U}(\mathbb{Z}[\sqrt{2}]) \Rightarrow & p=0, q=-2 s \\
\Rightarrow & \psi(\gamma)=(1+6 r)-6 s \sqrt{2}= \pm(1 \pm \sqrt{2})^{k},(k \in \mathbb{Z}) \\
\Rightarrow & (1+6 r)-6 s \sqrt{2}= \pm(17 \pm 12 \sqrt{2}),(k=4) \\
\Rightarrow & \gamma=-5-2 C_{1}-4 C_{3}-3 C_{4}+2 C_{5}-2 C_{7}+3 C_{8}+4 C_{9} \\
& \quad+2 C_{11}+6 a^{12},
\end{aligned}
$$




$$
\begin{aligned}
\text { ii }) \psi(\gamma) \in U(\mathbb{Z}[\sqrt{3}]) & \Rightarrow q=s=0 \\
& \Rightarrow \psi(\gamma)=(1+6 r)+2 p \sqrt{3}= \pm(2 \pm \sqrt{3})^{k},(k \in \mathbb{Z}) \\
& \Rightarrow(1+6 r)+2 p \sqrt{3}= \pm(7 \pm 4 \sqrt{3}),(k=2) \\
& \Rightarrow \gamma=3+2 C_{2}+C_{4}-C_{8}-2 C_{10}-2 a^{12}, \\
\text { iii }) \psi(\gamma) \in U(\mathbb{Z}[\sqrt{6}]) & \Rightarrow p=q=0 \\
& \Rightarrow \psi(\gamma)=(1+6 r)+2 s \sqrt{6}= \pm(5 \pm 2 \sqrt{6})^{k},(k \in \mathbb{Z}) \\
& \Rightarrow \psi(\gamma)=(1+6 r)+2 s \sqrt{6}= \pm 5 \pm 2 \sqrt{6},(k=1) \\
& \Rightarrow \gamma=-1+C_{1}-C_{4}+C_{5}-C_{7}+C_{8}-C_{11}+2 a^{12} .
\end{aligned}
$$

By considering Lemma 1 the generators can be written respectively as follows

$$
\begin{aligned}
& -5-2 C_{1}-4 C_{3}-3 C_{4}+2 C_{5}-2 C_{7}+3 C_{8}+4 C_{9}+2 C_{11}+3 C_{12}, \\
& \quad 3+2 C_{2}+C_{4}-C_{8}-2 C_{10}-C_{12}, \\
& -1+C_{1}-C_{4}+C_{5}-C_{7}+C_{8}-C_{11}+C_{12} .
\end{aligned}
$$

\section{Characterization of $V\left(\mathbb{Z} \mathbf{C}_{\mathbf{1 1}}^{+}\right)$and $V\left(\mathbb{Z} \mathbf{C}_{\mathbf{2 2}}^{+}\right)$}

Theorem 6. The normalized units of $V\left(\mathbb{Z} \mathbf{C}_{11}^{+}\right) \subset \mathbb{Z} \mathbf{C}_{11}$ are generated by the set

$$
V\left(\mathbb{Z} \mathbf{C}_{11}^{+}\right)=<-1+C_{1},-1+C_{2},-1-C_{3},-1+C_{4}>.
$$

Proof. Let $\gamma \in V\left(\mathbb{Z} \mathbf{C}_{11}^{+}\right)$be a generator of torsion-free unit. Then, we have

$$
\gamma=\gamma=\gamma_{0}+\sum_{i=1}^{5} \gamma_{i} C_{i}
$$

and

$$
\gamma_{0}+2 \gamma_{1}+2 \gamma_{2}+2 \gamma_{3}+2 \gamma_{4}+2 \gamma_{5}=1 \text {. }
$$

If we substitute $\gamma_{1}=p, \gamma_{2}=q, \gamma_{3}=r, \gamma_{4}=s, \gamma_{5}=t$ in (3.15), we can write

$$
\gamma_{0}=1-2(p+q+r+s+t) .
$$

On the other hand, if we denote $\omega=e^{\frac{2 \pi i}{11}}$ and $\alpha=\omega+\omega^{-1}$ then we can get the minimal polynomial of $\alpha$ over $\mathbb{Q}$ as follows;

$$
\min _{\mathbb{Q}}(\alpha, x)=x^{5}+x^{4}-4 x^{3}-3 x^{2}+3 x+1 .
$$

By regarding the following ring homomorphism:

$$
\psi: \begin{aligned}
\mathbb{Z} C_{11} & \rightarrow \mathbb{Z}[] \\
\sum \gamma_{i} a^{i} & \mapsto \sum \gamma_{i} \omega^{i}
\end{aligned}
$$


with equations (3.16) and (3.17), the image of the unit is

$$
\begin{aligned}
\psi(\gamma) & =\psi\left(\gamma_{0}+\sum_{i=1}^{5} \gamma_{i} C_{i}\right) \\
& =\gamma_{0}+\gamma_{1} \alpha+\gamma_{2}\left(\alpha^{2}-2\right)+\gamma_{3}\left(\alpha^{3}-3 \alpha\right)+\gamma_{4}\left(\alpha^{4}-4 \alpha^{2}+2\right) \\
& +\gamma_{5}\left(\alpha^{5}-5 \alpha^{3}+5 \alpha\right) \\
& =(1-2 p-4 q-2 r-3 t)+(p-3 r+2 t) \alpha+(q-4 s+3 t) \alpha^{2} \\
& +(r-t) \alpha^{3}+(s-t) \alpha^{4} .
\end{aligned}
$$

Since $\psi(\gamma) \in U(\mathbb{Z}[\alpha])=\left\{\sum_{i=0}^{4} a_{i} \alpha^{i}: \alpha^{5}+\alpha^{4}-4 \alpha^{3}-3 \alpha^{2}+3 \alpha+1=0\right\}$. By Table 2

$$
U(\mathbb{Z}[\alpha])=\left\langle\alpha, \alpha+1, \alpha^{2}-2, \alpha^{4}+\alpha^{3}-3 \alpha^{2}-3 \alpha\right\rangle .
$$

$\gamma \in U\left(\mathbb{Z}\left[a+a^{-1}\right]\right)$ can be obtained in the following single product of the fundamental units or their inverses;

$$
-\varepsilon_{1} \varepsilon_{2}^{-1} \varepsilon_{3} \varepsilon_{4}^{-1}, \varepsilon_{1}^{-1} \varepsilon_{2}^{-1} \varepsilon_{4}, \varepsilon_{1} \varepsilon_{4},-\varepsilon_{1}^{-1} \varepsilon_{2} \varepsilon_{3}^{-2} .
$$

Here

$$
\begin{array}{ll}
\psi(\gamma)=-\varepsilon_{1} \varepsilon_{2}^{-1} \varepsilon_{3} \varepsilon_{4}^{-1} & \Rightarrow \gamma=-1+C_{1} \\
\psi(\gamma)=\varepsilon_{1}^{-1} \varepsilon_{2}^{-1} \varepsilon_{4} & \Rightarrow \gamma=-1+C_{2} \\
\psi(\gamma)=\varepsilon_{1} \varepsilon_{4} & \Rightarrow \gamma=-1+C_{3} \\
\psi(\gamma)=-\varepsilon_{1}^{-1} \varepsilon_{2} \varepsilon_{3}^{-2} & \Rightarrow \gamma=-1+C_{4}
\end{array}
$$

\section{Corollary 3.}

$$
V\left(\mathbb{Z} \mathbf{C}_{22}^{+}\right)=<-1+C_{2},-1+C_{4},-1+C_{6},-1+C_{8}>.
$$

Proof. We obtain desired result by considering Theorem 6 with Remark 2 .

\section{ACKNOWLEDGEMENT}

The authors would like to thank Dr. Tevfik Bilgin for his helpful comments.

\section{REFERENCES}

[1] Z. Z. Aleev and G. A. Panina, "The units of cyclic groups of orders 7 and 9," Izv. Vyssh. Uchebn. Zaved. Mat., vol. 43, no. 11, pp. 81-84, 1999.

[2] V. s. Artamonov and A. s. Bovdi, "Integral group rings: groups of invertible elements and classical K-theory," Jour J. Soviet Math., vol. 57, no. 2, pp. 2931-2958, 1991.

[3] T. Bilgin, "Characterization of $u_{1}\left(\mathbb{Z} \mathbf{C}_{12}\right)$," Int. J. Pure Appl. Math., vol. 14, no. 4, pp. 531-535, 2004. 
[4] T. Bilgin, "A note on characterization of $n u(d n)$," Int. Electron. J. Algebra, vol. 3, pp. 135-140, 2008.

[5] D. B. Coleman, "On the modular group ring of a p-group," Proc. Amer. Math. Soc., vol. 15, pp. 511-514, 1964.

[6] M. Hertweck, "A counterexample to the isomorphism problem for integral group rings," Ann. of Math., vol. 154, no. 2, pp. 115-138, 2001

[7] M. Hertweck, "Contributions to the integral representation theory of groups," Ph.D. dissertation, University of Stuttgart, 2003.

[8] S. Jackowski and Z. Marciniak, "Group automorphisms inducing the identity map on cohomology," J. Pure Appl. Algebra, vol. 44, no. 1-3, pp. 241-250, 1987.

[9] E. Jespers, "Units in integral group rings: A survey, methods in ring theory," Lect. Notes Pure Appl. Math., vol. 198, pp. 141-169, 1998.

[10] G. Karpilovsky, Unit groups of group rings. New York: John Wiley and Sons Inc., 1989.

[11] W. Kimmerle and K. W. Roggenkamp, "Projective limits of group rings," J. Pure Appl. Algebra, vol. 88, no. 1-3, pp. 119-142, 1993.

[12] B. Kokluce and I. G. Kelebek, "A different characterization of $u_{1}\left(\mathbb{Z} \mathbf{C}_{7}\right)$ and $u_{1}\left(\mathbb{Z} \mathbf{C}_{9}\right)$," Int. J. Algebra, vol. 2, no. 15, pp. 701-706, 2008.

[13] Y. Li, "The normalizer of a metabelian group in its integral group ring," J. Algebra, vol. 256, pp. 343-351, 2002.

[14] Y. Li, M. M. Parmenter, and S. K. Sehgal, "On the normalizer property for integral group rings," Comm. Algebra, vol. 27, no. 9, pp. 4217-4223, 1999.

[15] Z. S. Marciniak and K. W. Roggenkamp, The normalizer of a finite group in its integral group ring and Cech cohomology, ser. NATO ASI Ser.II. Dordrecht: Kluwer Academic Publishers, 2001, vol. 28, ch. Algebra: Representation Theory, pp. 159-188.

[16] K. W. Roggenkamp and A. Zimmermann, "Outer group automorphisms may become inner in the integral group ring," J. Pure Appl. Algebra, vol. 103, no. 1, pp. 91-99, 1995.

[17] S. K. Sehgal, Units in integral group rings. New York: Marcel Dekker, 1993.

Authors' addresses

Bülent Köklüce

Mathematics Education, Faculty of Education, Fatih University, Istanbul, Turkey

E-mail address: bkokluce@fatih.edu.tr

\section{Nesibe Tüfekçi}

Department of Mathematics, Faculty of Arts and Sciences, Fatih University, Istanbul, Turkey

E-mail address: nesibe.tufekci@fatih.edu.tr 